\title{
TOF-SIMS Analysis with High Lateral and High Mass Resolution in Parallel
}

Felix Kollmer ${ }^{1}$, Nathan Havercroft ${ }^{2}$, Anja Henss ${ }^{3}$, Henrik Arlinghaus ${ }^{1}$, Wolfgang Paul ${ }^{1}$, Rudolf Moellers ${ }^{1}$, Ewald Niehuis ${ }^{1}$

1. IONTOF GmbH, Muenster, Germany

2. ION-TOF USA, Inc., Chestnut Ridge, NY, USA

3. Physikalisch-Chemisches Institut, Justus-Liebig University Giessen, Germany

Time-of-flight secondary ion mass spectrometry (TOF-SIMS) is a very sensitive surface analytical technique. It provides detailed elemental and molecular information about surfaces, thin layers, interfaces, and full three-dimensional analysis of the sample. A general strength of the applied time-offlight mass analyzer is the very high transmission that is due to the fact that the entire mass range is analyzed in parallel. Any selection of peaks prior to the analysis is not required.

However, the time-of-flight analyzer suffers from a trade-off between mass resolution, lateral resolution, and signal intensity. Even under low intensity conditions highest mass resolution and highest lateral resolution are not obtained at the same time. In our contribution, we will discuss an improved analyzer setup that allows us to overcome this limitation.

In order to obtain a high mass resolution a time-of-flight analyzer needs a very short start signal for the analysis cycle. Under static extraction conditions, a wider primary ion pulse broadens the width of any peak in the spectrum and thus reduces the achievable mass resolution. However, the generation of very short pulses by bunching inevitably broadens the spot size of the beam and leads to very low primary ion currents as well as long acquisition times. Even under lowest current conditions this approach remains a compromise since it does not allow one to obtain the maximum performance in terms of lateral resolution.

A promising approach in order to overcome this fundamental trade-off is to pulse the analyzer and to extract the secondary ions just after the primary beam hits the surface (delayed extraction). Under delayed extraction conditions, the width of the primary ion pulse no longer influences the uncertainty of the start signal of the time-of-flight analysis. High lateral resolution and high mass resolution are obtained in parallel with long ion pulses at higher, and thus more useful, ion currents. Moreover, the delayed extraction mode does improve the analysis of topographic surfaces in terms of transmission and mass resolution. Although this approach has been well known for many years and is frequently applied in MALDI, the application in TOF-SIMS is only recently increasing [1]. We have optimized this mode and we will show that the achieved improvements are very useful for the analysis of most kinds of samples and analytical tasks and that this mode has the potential to become a standard mode for TOFSIMS analyses as well.

In our contribution, we will discuss the pros and cons of the delayed extraction mode and we will show how to optimize the analysis parameters and the operation. Investigated samples include: pharmaceuticals, biological samples (figure 1), reference samples, and deep FIB-milled crater walls. The latter are especially demanding with respect to transmission and mass resolution because of the very strong vertical surface topography. 
References:

[1] Quentin P. Vanbellingen, et al, Rapid Commun. Mass Spectrom. 29 (2015), p. 1187, DOI:

$10.1002 / \mathrm{rcm} .7210$

[2] Anja Worrich et al, Nat. Commun. 8 (2017), p. 15472, DOI: 10.1038/NCOMMS1547
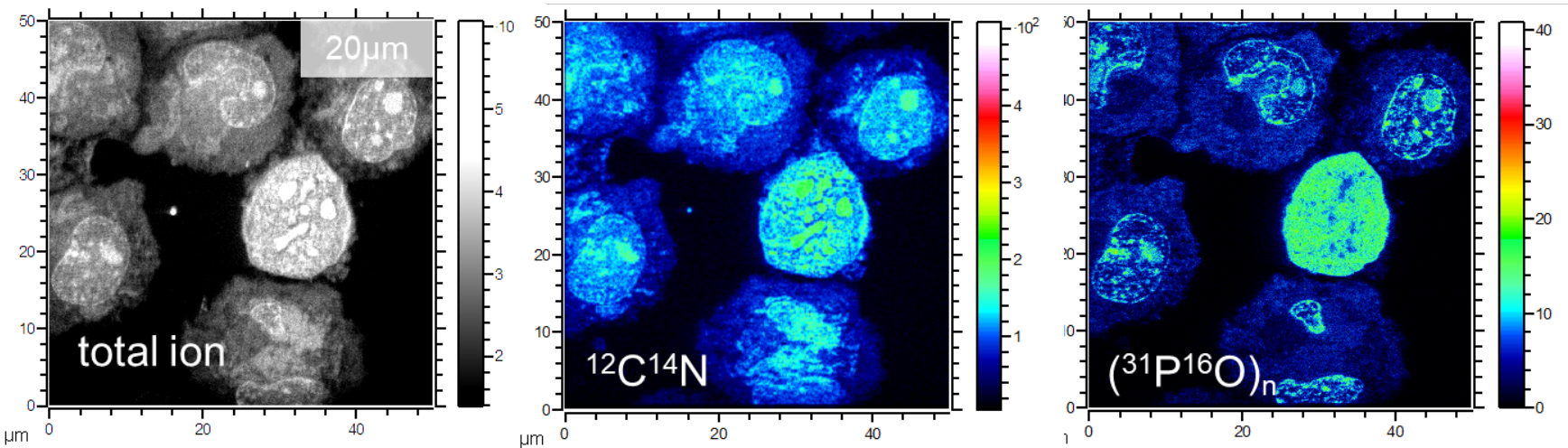

Figure 1. TOF-SIMS analysis of bortezomib treated myeloma cells. 